\title{
C-reactive protein is associated with severity of thought and language dysfunction in patients with schizophrenia
}

This article was published in the following Dove Press journal:

Neuropsychiatric Disease and Treatment

\author{
Chun-Hung Chang (iD ${ }^{1-3}$ \\ Hsien-Yuan Lane (iD) $1,2,4,5, *$ \\ Chieh-Yu Liu iD ${ }^{6}$ \\ Po-Chih Cheng ${ }^{6}$ \\ Shaw-ji Chen (iD ${ }^{7,8}$ \\ Chieh-Hsin Lin (D) $1,4,9, *$
}

'Institute of Clinical Medical Science, China Medical University, Taichung,

Taiwan; ${ }^{2}$ Department of Psychiatry \& Brain Disease Research Center, China Medical University Hospital, Taichung, Taiwan; ${ }^{3}$ An Nan Hospital, China Medical University, Tainan, Taiwan; ${ }^{4}$ Graduate Institute of Biomedical Sciences, China Medical University, Taichung, Taiwan; ${ }^{5}$ Department of Psychology, College of Medical and Health Sciences, Asia University, Taichung, Taiwan;

${ }^{6}$ Biostatistical Consulting Laboratory, Department of Speech Language Pathology and Audiology, National Taipei University of Nursing and Health Sciences, Taipei, Taiwan; ${ }^{7}$ Department of Psychiatry, Mackay Memorial Hospital

Taitung Branch, Taitung, Taiwan;

${ }^{8}$ Department of Medicine, Mackay Medical College, New Taipei, Taiwan;

${ }^{9}$ Kaohsiung Chang Gung Memorial Hospital, Chang Gung University College of Medicine, Kaohsiung, Taiwan

*These authors contributed equally to this work

Correspondence: Hsien-Yuan Lane Department of Psychiatry, China Medical University Hospital, No. 2, Yuh-Der Road, Taichung 404, Taiwan

Email hylane@gmail.com

Chieh-Hsin Lin

Department of Psychiatry, Kaohsiung

Chang Gung Memorial Hospital, No 123 ,

Dapi Road, Niaosong District, Kaohsiung

833, Taiwan

Tel +886 773 I 7123 Ext 8753

Fax +8867732 68I7

Email cyndi36@gmail.com
Background: Numerous studies have demonstrated an association between C-reactive protein (CRP) levels and schizophrenia. However, the findings on psychotic severity and cognition remain inconsistent. The relationship between CRP and formal thought disorder in subdomains remains unclear.

Methods: We enrolled stable patients (defined as those who had no treatment changes during the 4-week period before evaluation) with a diagnosis of schizophrenia or schizoaffective disorder, according to the Diagnostic and Statistical Manual of Mental Disorders, Fifth Edition. We used the 30-item Thought and Language Disorder (TALD) scale to evaluate thought and language dysfunction over four subscales. We assessed psychotic symptoms using the Positive and Negative Syndrome Scale (PANSS). We collected fasting venous blood and measured plasma CRP levels.

Results: We enrolled 60 patients with schizophrenia. All patients received TALD and PANSS evaluation, and 33 of them had their CRP levels checked. The multivariate regression analysis indicated that CRP levels were significantly associated with the total score on the TALD ( $\mathrm{t}=2.757, P=0.010)$ and the TALD Objective Positive subscale $(\mathrm{t}=2.749, P=0.011)$, after sex, age, duration of illness (in years), and use of atypical antipsychotics were adjusted for. Additionally, CRP was significantly associated with the PANSS positive subscale $(\mathrm{t}=2.102, P=0.045)$. A significantly positive correlation was observed between the total scores on the TALD scale and PANSS $(\rho=0.751, P<0.001)$.

Conclusion: Our results suggest that abnormal CRP levels are significantly associated with formal thought and language dysfunction in the Objective Positive subdomain and positive psychotic symptoms.

Keywords: C-reactive protein, formal thought disorder, schizophrenia

\section{Introduction}

C-reactive protein (CRP) is the primary inflammation biomarker in clinical practice. ${ }^{1}$ Numerous studies have identified an association between chronic inflammation and schizophrenia., ${ }^{2,3}$ A meta-analysis included 26 longitudinal or crosssectional trials and reported moderately increased CRP levels in patients with schizophrenia irrespective of antipsychotic use. ${ }^{2}$ The CRP levels were also reported to increase with the severity of positive symptoms but not with negative symptoms. ${ }^{2}$ By contrast, other studies have demonstrated that abnormally elevated CRP levels are not associated with the severity of psychotic symptoms. ${ }^{4-8}$ Although inconsistent, these findings suggest that CRP is associated with positive but not 
negative symptoms; these effects may be confounded by factors such as different psychotic phases (eg, acute or chronic phase). ${ }^{9}$ For instance, three trials with community-dwelling stabilized outpatients observed no association between elevated CRP and psychotic severity. $6,10,11$

Many studies have suggested that high CRP levels are also associated with varying levels of cognitive performance impairment in patients with schizophrenia., $4,12-14$ Moreover, abnormally elevated CRP levels are associated with different cognitive function impairments, including impairments in short-term memory, ${ }^{15}$ abstract reasoning, working memory, learning ability, semantic memory, mental flexibility, visual attention, and processing speed. ${ }^{14}$ However, other studies have observed no significant correlations between abnormal CRP levels and cognitive functions. ${ }^{16-18}$ Boozalis et al observed that CRP was correlated with negative symptoms but not cognitive function. ${ }^{18}$

Formal thought disorder (FTD), a core schizophrenia symptom, is characterized by disorganized speech and a deficit in the ability to organize thought; FTD results from the inappropriate use of aspects of language (particularly, semantics). Previous studies have demonstrated that FTD is a marker of psychotic severity ${ }^{19,20}$ and impaired cognition in schizophrenia. ${ }^{21} \mathrm{~A}$ recent meta-analysis reported that neurocognitive and linguistic capabilities are correlated with positive and negative FTD. ${ }^{22}$ However, the relationship between CRP and FTD remains unclear. Moreover, a study recommended objective and subjective FTD assessment in addition to the assessment of positive and negative subdomains. ${ }^{23}$ On the basis of these studies, we hypothesize that CRP is associated with FTD in different subdomains. Therefore, in this study, we investigated the relationship between CRP levels and the 30-item Thought and Language Disorder (TALD) scale scores. We studied the objective and subjective subdomains as well as the positive and negative subdomains in patients with schizophrenia.

\section{Methods}

\section{Study population}

The Institutional Review Board of China Medical University Hospital approved this study (CMUH107REC3-055). We followed the Declaration of Helsinki recommendations. In this study, all participants provided written informed consent.

We recruited patients, from a chronic ward and an outpatient clinic, with a diagnosis of either schizophrenia or schizoaffective disorder. We obtained demographic data on age, sex, age of onset, duration of illness, years of education, and body mass index (BMI).

\section{Inclusion criteria}

We enrolled stable patients (defined as patients with no treatment changes during a 4-week period before evaluation) with the diagnosis of schizophrenia or schizoaffective disorder, according to the Diagnostic and Statistical Manual of Mental Disorders, Fifth Edition. Diagnosis was confirmed by trained psychiatrists.

\section{Exclusion criteria}

We excluded patients with a history of neurological disorders, including stroke, epilepsy, and the effects of head injury. Patients with any current major infectious or inflammatory diseases, particularly autoimmune diseases such as lupus and rheumatoid arthritis, were excluded. We also excluded patients who used corticosteroids or nonsteroidal anti-inflammatory drugs.

\section{Sample collection and CRP measurement}

We collected fasting venous blood and measured plasma CRP levels. In this study, we used a particle-enhanced immunoturbidimetric assay (IMMULITE1; Diagnostic Products Corp., Los Angeles, California, USA) to measure high-sensitivity CRP. The coefficient of variation was $5 \%$ at $0.02 \mathrm{mg} / \mathrm{dL} \mathrm{CRP}$.

\section{Thought language function and psychotic symptoms}

We used the 30 -item TALD scale ${ }^{23}$ to evaluate thought and language dysfunction. The TALD scale comprises four subscales: Objective Positive, Objective Negative, Subjective Positive, and Subjective Negative. We used the Positive and Negative Syndrome Scale (PANSS) to assess psychotic symptoms. ${ }^{24}$ The PANSS comprises three subscales: positive, negative, and general.

\section{Statistical analysis}

We provided descriptive statistics of the mean, median and standard deviation. A multivariate regression analysis was used to evaluate the associations between the CRP and TALD scale scores and between the CRP and the PANSS scores. We measured the correlation between the TALD scale and PANSS and calculated Spearman's rank correlation. We performed the analyses using IBM SPSS, version 
20 (IBM Corp., Armonk, NY, USA), and statistical significance was defined as a two-tailed $P<0.05$.

\section{Results}

Our sample comprised 60 patients with schizophrenia. The demographic and clinical characteristics of the sample are detailed in Table 1 . There were $51.7 \%$ men in the sample population. The mean age of the patients was $44.78 \pm 9.88$ years, and the mean age during schizophrenia

Table I Clinical and demographic characteristics of individuals with schizophrenia $(\mathrm{N}=60)$

\begin{tabular}{|l|l|l|}
\hline & Mean or N (\%) & S.D. \\
\hline Age & $44.78(9.877)$ & \\
Gender, male & $31(51.7 \%)$ & \\
Education & 11.18 & 2.891 \\
Age of onset & 25.4 & 8.166 \\
Illness duration (years) & 19.38 & 8.019 \\
PANSS Positive & 16.55 & 2.594 \\
PANSS Negative & 17.48 & 1.855 \\
PANSS General & 35.95 & 4.276 \\
PANSS Total & 69.98 & 7.019 \\
TALD Objective Positive & 13.85 & 9.231 \\
TALD Objective & 3.00 & 2.731 \\
Negative & & \\
TALD Subjective Positive & 1.98 & 1.996 \\
TALD Subjective & 9.70 & 5.036 \\
Negative & & \\
TALD Total & 28.53 & \\
First generation & $16(26.67 \%)$ & \\
antipsychotics & & \\
\hline Second generation & $52(86.67 \%)$ & \\
antipsychotics & & \\
Olanzapine & $9(15.0 \%)$ & \\
Aripiprazole & $9(15.0 \%)$ & \\
Risperidone & $9(15.0 \%)$ & \\
Quetiapine & $8(13.3 \%)$ & \\
Paliperidone & $7(11.7 \%)$ & \\
Clozapine & $7(11.7 \%)$ & \\
Amisulpride & $5(8.3 \%)$ & \\
Zotepine & $2(3.3 \%)$ & \\
\hline DM & $6(10.0 \%)$ & \\
Hypertension & $12(20.0 \%)$ & \\
BMI & 0.405 & \\
CRP & $-14[0.86 \pm 1.32$ & \\
CRP, mg/dL & & \\
CRP level after log- & \\
transformation & & \\
\hline Abbrevitions & \\
\hline
\end{tabular}

Abbreviations: PANSS, Positive and Negative Syndrome Scale; TALD, Thought and Language Disorder Scale; DM, diabetes mellitus; BMI, body mass index; CRP, Creactive protein. onset was $25.40 \pm 8.17$ years. The mean duration of illness was $19.38 \pm 8.019$ years, and the mean PANSS total score was $69.98 \pm 7.02$. Among the 60 subjects, 57 patients were diagnosed as having schizophrenia and 3 as having schizoaffective disorder. All patients were interviewed with the TALD scale and received a PANSS evaluation, and 33 of them had their CRP levels checked. All patients were receiving antipsychotic treatment when they were enrolled. The major antipsychotics used were secondgeneration antipsychotics (in $52 / 60$ or $86.67 \%$ of patients), and the three leading second-generation antipsychotics were olanzapine, aripiprazole, and risperidone. Among the 60 patients, one was enrolled from an outpatient clinic, and the other 59, from a chronic ward.

\section{CRP and thought and language dysfunction}

The multivariate regression analysis indicated that CRP levels were significantly associated with scores on the TALD scale $(\mathrm{t}=2.757, P=0.010)$, after sex, age, duration of illness (in years), and use of atypical antipsychotics were adjusted for. Additionally, in the analysis of the four TALD subscales, CRP was significantly associated with the Objective Positive subscale of the TALD ( $\mathrm{t}=2.749, P=0.011$ ), but associations with the other three TALD subscales were nonsignificant (Table 2).

\section{CRP and psychotic symptoms}

In this study, CRP levels were associated with PANSS scores, but the association was nonsignificant $(\mathrm{t}=1.744$, $P=0.092$ ), after sex, age, duration of illness (in years), and use of atypical antipsychotics were adjusted for. Additionally, in the analysis of the four PANSS subscales, CRP was significantly associated with the positive subscale of the PANSS ( $\mathrm{t}=2.102, P=0.045)$, but the association with the other two PANSS subscales was nonsignificant (Table 2).

\section{Thought, language dysfunction and psychotic symptoms}

A significantly positive correlation was observed between the total score on the TALD and PANSS total score (rho=0.751, $P<0.001$; Figure 1). In the subscale analysis, the TALD Objective Positive subscale was significantly correlated with three subscales of the PANSS $(\rho=0.481$, 0.540 , and 0.585 , respectively, $P<0.001)$. The TALD Subjective Positive subscale was significantly correlated 


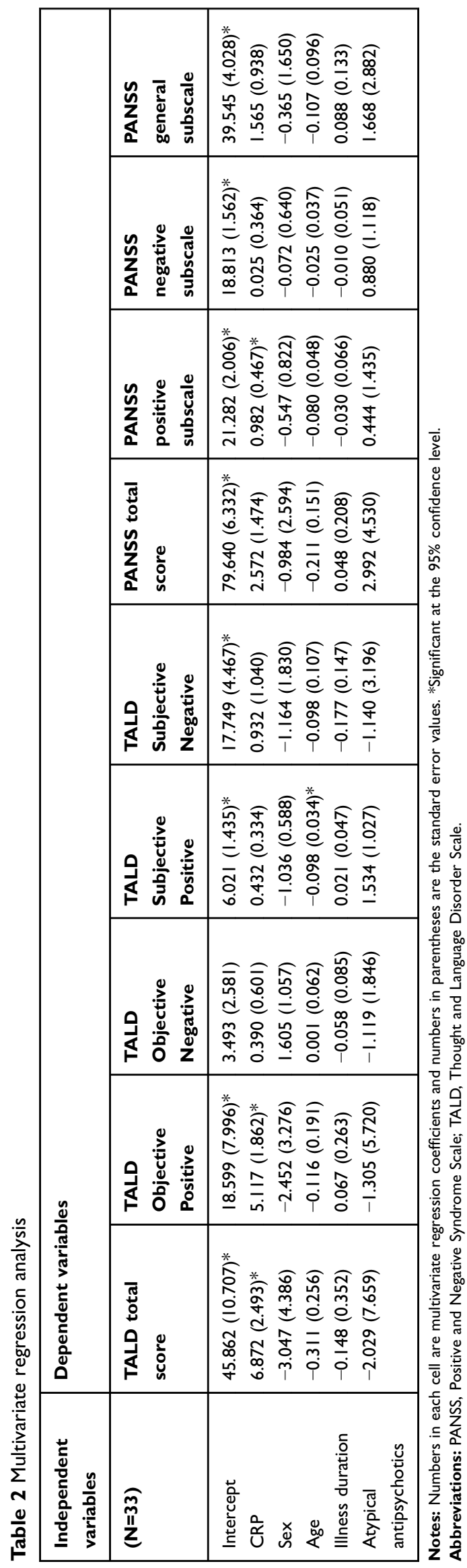

with the PANSS positive subscale $(\rho=0.264, P=0.042)$, and the TALD Subjective Negative subscale was significantly correlated with both the PANSS positive and negative subscales $(\rho=0.528, P<0.001 ; \rho=0.335, P=0.009$; Table 3).

\section{Discussion}

To our knowledge, this is the first study to investigate the association between CRP and FTD. The findings of this study are as follows: (a) CRP levels are significantly associated with the total scores on the TALD and the TALD Objective Positive subscale, (b) CRP levels are significantly associated with the PANSS positive subscale, and (c) the total score on the TALD and some TALD subscale scores are significantly correlated with all scores on the PANSS.

In this study, the multivariate regression analysis indicated that CRP levels are significantly associated with the total score on the TALD $(\mathrm{t}=2.757, P=0.010)$ after sex, age, duration of illness (in years), and atypical antipsychotic use were adjusted for. Furthermore, in the analysis of the four TALD subscales, CRP was significantly associated with the TALD Objective Positive subscale $(\mathrm{t}=2.749$, $P=0.011$ ). This is the first study to investigate the association between CRP and FTD in the four subscales. Kircher and his colleagues invented a comprehensive assessment tool, the TALD scale, ${ }^{23}$ to evaluate four FTD subdomains: Objective Positive, Objective Negative, Subjective Positive, and Subjective Negative. Earlier FTD rating scales were in the descriptive psychopathological tradition. ${ }^{25,26}$ Furthermore, we observed a significantly positive correlation between the total score on the TALD and PANSS total score $(\rho=0.751, P<0.001$; Figure 1$)$. In subscale analysis, the TALD Objective Positive subscale score was significantly correlated with three PANSS subscales ( $\rho=0.481,0.540$, and 0.585 , all $P<0.001$; Table 3 ). Our findings agree with the results of Kircher and his colleagues, who observed a correlation between the TALD Objective Positive subscale and the Scale for the Assessment of Positive Symptoms (SAPS; $\rho=0.925$, $P<0.01) .{ }^{23}$ A study that examined 51 patients with schizophrenia also reported a significant correlation between the TALD Objective Positive subscale score and executive dysfunctions. ${ }^{27}$ Further investigation may be needed on the relationships between CRP, FTD, and cognition.

In this study, CRP was significantly associated with the PANSS positive subscale $(\mathrm{t}=2.102, P=0.045)$, although CRP's associations with the PANSS total score and the 


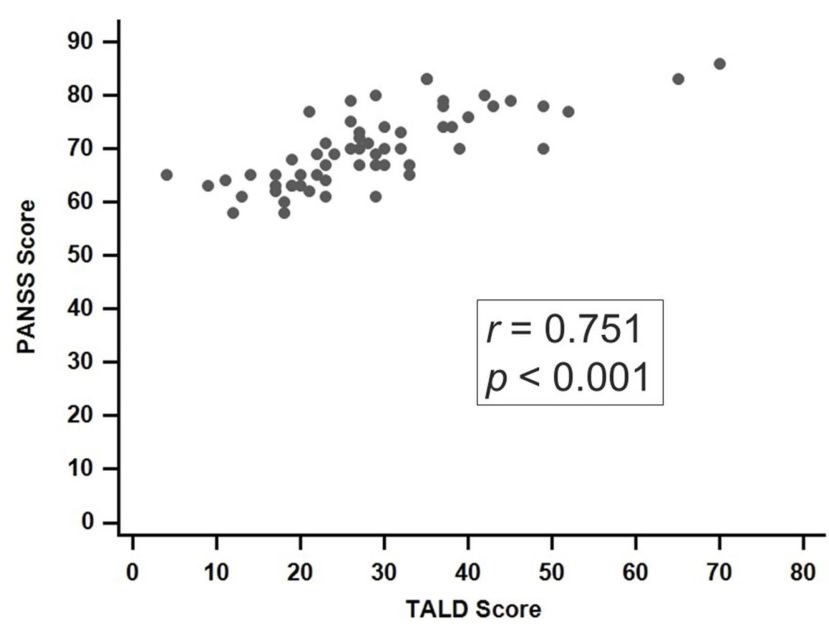

Figure I Scatter plots and correlation analysis between the TALD scale and PANSS.

Abbreviations: PANSS, Positive and Negative Syndrome Scale; TALD, Thought and Language Disorder Scale.

two PANSS subscales of negative and general were nonsignificant. Our findings agree with a previous metaanalysis $^{2}$ in which meta-regression analyses suggested that the PANSS positive subscale was positively related to CRP levels (slope $=0.12,95 \% \mathrm{CI}=0.03-0.23, P=0.013$ ), but the association between the PANSS total score and PANSS negative subscale was nonsignificant. A study on 26 patients with either schizophrenia or schizoaffective disorder reported that patients with greater CRP levels had a significantly greater PANSS total score, negative subscale score, and general subscale score relative to patients with normal CRP levels ( $P=0.017,0.016$, and 0.014 , respectively). ${ }^{28}$ Another study whose sample comprised 48 patients with first-episode psychosis and 74 patients with schizophrenia in an acute exacerbation phase observed a positive correlation between high-sensitivity CRP levels and the severity of positive symptoms $(\rho=0.494, P=0.004){ }^{29}$ Two studies have observed that CRP is correlated with negative symptoms. ${ }^{18,30}$ However, some studies have observed no significant correlation between abnormal CRP levels and psychotic severity. ${ }^{4,6,8}$
These discrepancies warrant further meta-analyses, trials with larger sample sizes, and comprehensive analyses of confounding factors to investigate the subgroup that is patients with severe psychosis.

We propose two reasons that CRP is associated with psychotic positive symptoms and impaired cognition: First, increased CRP may indirectly cause hypofunction of the Nmethyl-D-aspartate (NMDA) receptor pathway that plays a crucial role in schizophrenia and cognition. ${ }^{31,32}$ Studies have observed that peripheral CRP can increase the permeability of the blood-brain barrier by influencing the tight junction $^{33}$ that plays a role in psychiatric disorders. ${ }^{34,35}$ After entering the brain, CRP can induce an inflammatory state in the microglia, ${ }^{36}$ which then releases interleukin (IL)-6 and transforming growth factor $\beta .^{36,37}$ This induces the astrocytes to metabolize tryptophan into kynurenic acid, which may result in hypoglutamatergic function because it acts as an antagonist to NMDA receptors as well as dopaminergic hyperfunction in the limbic system. These functions are involved in the development of positive psychotic symptoms in schizophrenia. ${ }^{38-41}$ Second, CRP may act as a proxy for IL-6, correlated with positive psychotic symptoms. $^{37,42}$

\section{Limitations}

This study has several limitations. First, we did not assess the cognitive functions using standard tools. Standard cognitive measurements should be used to evaluate the relationships between CRP, cognitive functions, and thought dysfunction in future studies. Second, we only evaluated CRP; other inflammatory biomarkers such as IL-6 were not evaluated. The results pertaining to CRP levels were based on only 33 patients. Further investigation should be conducted with a larger sample of patients in different psychotic phases. Third, additional factors, such as alcohol and substance abuse, were not included in our analysis. Furthermore, to confirm the diagnoses of the patients, we recommend further trials that use standardized clinical

Table 3 Correlations between the four subdomains of the TALD scale and three subdomains of the PANSS

\begin{tabular}{|l|l|l|l|l|}
\hline \multirow{2}{*}{ PANSS three subdomains } & \multicolumn{4}{l|}{ TALD four subdomains } \\
\cline { 2 - 5 } & Objective Positive & Objective Negative & Subjective Positive & Subjective Negative \\
\hline Positive & $0.481^{* *}$ & 0.206 & $0.264^{*}$ & $0.528^{* *}$ \\
Negative & $0.540^{* *}$ & 0.080 & 0.062 & $0.335^{* *}$ \\
General & $0.585^{* *}$ & 0.250 & 0.193 & 0.189 \\
\hline
\end{tabular}

Notes: **Correlation is significant at the 0.01 level (2-tailed). *Correlation is significant at the 0.05 level (2-tailed).

Abbreviations: PANSS, Positive and Negative Syndrome Scale; TALD, Thought and Language Disorder Scale. 
examination methods such as the Structured Clinical Interview for Mental Disorders.

\section{Conclusion}

CRP was significantly associated with formal thought and language dysfunction in the subgroup of patients that score highly on the TALD Objective Positive subscale and have positive psychotic symptoms.

\section{Acknowledgements}

This work was supported by grants from Ministry of Science and Technology, Taiwan (MOST 108-2314-B039-002), National Health Research Institutes (NHRIEX108-1073NI), China Medical University, Taiwan (CMU107-BC-4), and Taiwan Ministry of Health and Welfare Clinical Trial and Research Center of Excellence (MOHW108-TDU-B-212-133004).

\section{Disclosure}

The authors report no conflicts of interest in this work.

\section{References}

1. Lopresti AL, Maker GL, Hood SD, Drummond PD. A review of peripheral biomarkers in major depression: the potential of inflammatory and oxidative stress biomarkers. Prog Neuropsychopharmacol Biol Psychiatry. 2014;48:102-111. doi:10.1016/j.pnpbp.2013.09.017

2. Fernandes BS, Steiner J, Bernstein HG, et al. C-reactive protein is increased in schizophrenia but is not altered by antipsychotics: metaanalysis and implications. Mol Psychiatry. 2016;21(4):554-564. doi:10.1038/mp.2015.87

3. Fond G, d'Albis MA, Jamain S, et al. The promise of biological markers for treatment response in first-episode psychosis: a systematic review. Schizophr Bull. 2015;41(3):559-573. doi:10.1093/schbul/ sbv002

4. Dickerson F, Stallings C, Origoni A, Boronow J, Yolken R. C-reactive protein is associated with the severity of cognitive impairment but not of psychiatric symptoms in individuals with schizophrenia. Schizophr Res. 2007;93(1-3):261-265. doi:10.1016/j.schres.2007.03.022

5. Faugere M, Micoulaud-Franchi JA, Alessandrini M, et al. Quality of life is associated with chronic inflammation in schizophrenia: a crosssectional study. Sci Rep. 2015;5:10793. doi:10.1038/srep10793

6. Fond G, Godin O, Brunel L, et al. Peripheral sub-inflammation is associated with antidepressant consumption in schizophrenia. Results from the multi-center FACE-SZ data set. $J$ Affect Disord. 2016;191:209-215. doi:10.1016/j.jad.2015.11.017

7. Frydecka D, Misiak B, Pawlak-Adamska E, et al. Interleukin-6: the missing element of the neurocognitive deterioration in schizophrenia? The focus on genetic underpinnings, cognitive impairment and clinical manifestation. Eur Arch Psychiatry Clin Neurosci. 2015;265(6):449459. doi:10.1007/s00406-014-0533-5

8. Micoulaud-Franchi JA, Faugere M, Boyer L, et al. Elevated C-reactive protein is associated with sensory gating deficit in schizophrenia. Schizophr Res. 2015;165(1):94-96. doi:10.1016/j.schres.2015.03.018

9. Fond G, Lancon C, Auquier P, Boyer L. C-Reactive protein as a peripheral biomarker in schizophrenia. An updated systematic review. Front Psychiatry. 2018;9:392. doi:10.3389/fpsyt.2018.00392
10. Dickerson F, Stallings C, Origoni A, et al. C-reactive protein is elevated in schizophrenia. Schizophr Res. 2013;143(1):198-202. doi:10.1016/j.schres.2012.10.041

11. Fond G, Resseguier N, Schurhoff F, et al. Relationships between low-grade peripheral inflammation and psychotropic drugs in schizophrenia: results from the national FACE-SZ cohort. Eur Arch Psychiatry Clin Neurosci. 2018;268(6):541-553. doi:10.1007/ s00406-017-0847-1

12. Misiak B, Stanczykiewicz B, Kotowicz K, Rybakowski JK, Samochowiec J, Frydecka D. Cytokines and C-reactive protein alterations with respect to cognitive impairment in schizophrenia and bipolar disorder: a systematic review. Schizophr Res. 2018;192:1629. doi:10.1016/j.schres.2017.04.015

13. Dickerson F, Stallings C, Origoni A, Vaughan C, Khushalani S, Yolken R. Additive effects of elevated C-reactive protein and exposure to herpes simplex virus type 1 on cognitive impairment in individuals with schizophrenia. Schizophr Res. 2012;134(1):83-88. doi:10.1016/j.schres.2011.10.003

14. Bulzacka E, Boyer L, Schurhoff F, et al. Chronic peripheral inflammation is associated with cognitive impairment in schizophrenia: results from the multicentric FACE-SZ dataset. Schizophr Bull. 2016;42(5):1290-1302. doi:10.1093/schbul/sbw029

15. Dorofeikova M, Neznanov N, Petrova N. Cognitive deficit in patients with paranoid schizophrenia: its clinical and laboratory correlates. Psychiatry Res. 2018;262:542-548. doi:10.1016/j.psychres.2017.09.041

16. Hope S, Hoseth E, Dieset I, et al. Inflammatory markers are associated with general cognitive abilities in schizophrenia and bipolar disorder patients and healthy controls. Schizophr Res. 2015;165(23):188-194. doi:10.1016/j.schres.2015.04.004

17. Joseph J, Depp C, Martin AS, et al. Associations of high sensitivity Creactive protein levels in schizophrenia and comparison groups. Schizophr Res. 2015;168(1-2):456-460. doi:10.1016/j.schres.2015.08.019

18. Boozalis T, Teixeira AL, Cho RY, Okusaga O. C-Reactive protein correlates with negative symptoms in patients with schizophrenia. Front Public Health. 2017;5:360. doi:10.3389/fpubh.2017.00081

19. Roche E, Creed L, MacMahon D, Brennan D, Clarke M. The epidemiology and associated phenomenology of formal thought disorder: a systematic review. Schizophr Bull. 2015;41(4):951-962. doi:10.1093/ schbul/sbu129

20. Roche E, Lyne JP, O'Donoghue B, et al. The factor structure and clinical utility of formal thought disorder in first episode psychosis. Schizophr Res. 2015;168(1-2):92-98. doi:10.1016/j.schres.2015.07.049

21. Kerns JG, Berenbaum H. Cognitive impairments associated with formal thought disorder in people with schizophrenia. $J$ Abnorm Psychol. 2002;111(2):211-224.

22. Bora E, Yalincetin B, Akdede BB, Alptekin K. Neurocognitive and linguistic correlates of positive and negative formal thought disorder: a meta-analysis. Schizophr Res. 2019. doi:10.1016/j.schres.2019.05.025

23. Kircher T, Krug A, Stratmann M, et al. A rating scale for the assessment of objective and subjective formal Thought and Language Disorder (TALD). Schizophr Res. 2014;160(1-3):216221. doi:10.1016/j.schres.2014.10.024

24. Kay SR, Fiszbein A, Opler LA. The positive and negative syndrome scale (PANSS) for schizophrenia. Schizophr Bull. 1987;13(2):261276. doi:10.1093/schbul/13.2.261

25. Andreasen NC. Scale for the assessment of thought, language, and communication (TLC). Schizophr Bull. 1986;12(3):473-482. doi: $10.1093 / \mathrm{schbul} / 12.3 .473$

26. Andreasen NC, Grove WM. Thought, language, and communication in schizophrenia: diagnosis and prognosis. Schizophr Bull. 1986;12 (3):348-359. doi:10.1093/schbul/12.3.348

27. Nagels A, Fahrmann P, Stratmann M, et al. Distinct neuropsychological correlates in positive and negative formal thought disorder syndromes: the thought and language disorder scale in endogenous psychoses. Neuropsychobiology. 2016;73(3):139-147. doi:10.1159/ 000441657 
28. Fan X, Pristach C, Liu EY, Freudenreich O, Henderson DC, Goff DC. Elevated serum levels of C-reactive protein are associated with more severe psychopathology in a subgroup of patients with schizophrenia. Psychiatry Res. 2007;149(1-3):267-271. doi:10.1016/j.psychres. 2006.07.011

29. Bolu A, Aydin MS, Akgun A, et al. Serum levels of high sensitivity C-reactive protein in drug-naive first-episode psychosis and acute exacerbation of schizophrenia. Clin Psychopharmacol Neurosci. 2019;17(2):244-249. doi:10.9758/cpn.2019.17.2.244

30. Fawzi MH, Fawzi MM, Fawzi MM, Said NS. C-reactive protein serum level in drug-free male Egyptian patients with schizophrenia. Psychiatry Res. 2011;190(1):91-97. doi:10.1016/j.psychres.2011.05.010

31. Lin CH, Lane HY, Tsai GE. Glutamate signaling in the pathophysiology and therapy of schizophrenia. Pharmacol Biochem Behav. 2012;100(4):665-677. doi:10.1016/j.pbb.2011.03.023

32. Li F, Tsien JZ. Memory and the NMDA receptors. $N$ Engl J Med. 2009;361(3):302-303. doi:10.1056/NEJMcibr0902052

33. Campbell BM, Charych E, Lee AW, Moller T. Kynurenines in CNS disease: regulation by inflammatory cytokines. Front Neurosci. 2014;8:12. doi:10.3389/fnins.2014.00012

34. Najjar S, Pearlman DM, Devinsky O, Najjar A, Zagzag D. Neurovascular unit dysfunction with blood-brain barrier hyperpermeability contributes to major depressive disorder: a review of clinical and experimental evidence. J Neuroinflammation. 2013;10:142. doi:10.1186/1742-2094-10-151

35. Uranova NA, Zimina IS, Vikhreva OV, Krukov NO, Rachmanova VI, Orlovskaya DD. Ultrastructural damage of capillaries in the neocortex in schizophrenia. World J Biol Psychiatry. 2010;11(3):567-578. doi: $10.3109 / 15622970903414188$
36. Adami C, Sorci G, Blasi E, Agneletti AL, Bistoni F, Donato R. S100B expression in and effects on microglia. Glia. 2001;33 (2):131-142.

37. Schwieler L, Larsson MK, Skogh E, et al. Increased levels of IL-6 in the cerebrospinal fluid of patients with chronic schizophrenia-significance for activation of the kynurenine pathway. J Psychiatry Neurosci. 2015;40(2):126-133

38. Kegel ME, Bhat M, Skogh E, et al. Imbalanced kynurenine pathway in schizophrenia. Int J Tryptophan Res. 2014;7:15-22. doi:10.4137/ IJTR.S16800

39. Koshy Cherian A, Gritton H, Johnson DE, Young D, Kozak R, Sarter M. A systemically-available kynurenine aminotransferase II (KAT II) inhibitor restores nicotine-evoked glutamatergic activity in the cortex of rats. Neuropharmacology. 2014;82:41-48. doi:10.1016/j.neuropharm.2014. 03.004

40. Schwarcz R, Bruno JP, Muchowski PJ, Wu HQ. Kynurenines in the mammalian brain: when physiology meets pathology. Nat Rev Neurosci. 2012;13(7):465-477. doi:10.1038/nrn3257

41. Wu HQ, Okuyama M, Kajii Y, Pocivavsek A, Bruno JP, Schwarcz R. Targeting kynurenine aminotransferase II in psychiatric diseases: promising effects of an orally active enzyme inhibitor. Schizophr Bull. 2014;40(Suppl 2):S152-S158. doi:10.10 93/schbul/sbt157

42. Magalhaes CA, Ferreira CN, Loures CMG, et al. Leptin, hsCRP, TNF-alpha and IL-6 levels from normal aging to dementia: relationship with cognitive and functional status. $J$ Clin Neurosci. 2018;56:150-155. doi:10.1016/j.jocn.2018.08.027
Neuropsychiatric Disease and Treatment

\section{Publish your work in this journal}

Neuropsychiatric Disease and Treatment is an international, peerreviewed journal of clinical therapeutics and pharmacology focusing on concise rapid reporting of clinical or pre-clinical studies on a range of neuropsychiatric and neurological disorders. This journal is indexed on PubMed Central, the 'PsycINFO' database and CAS, and is the official journal of The International Neuropsychiatric Association (INA). The manuscript management system is completely online and includes a very quick and fair peer-review system, which is all easy to use. Visit http://www.dovepress.com/testimonials.php to read real quotes from published authors. 